\title{
De l'observation vidéo à l'observation in situ du travail enseignant en milieu difficile: étude des effets sur des professeurs stagiaires
}

\section{Luc Ria, Guillaume Serres et Serge Leblanc}

Cet article décrit et analyse les effets d'un dispositif d'observation destiné aux professeurs stagiaires de l'enseignement secondaire d'un institut de formation en France ayant pour vocation de les préparer à leur première affectation professionnelle. Confrontés successivement à une série d'extraits vidéo sur le travail enseignant en milieu difficile, puis deux mois plus tard au travail en situation réelle de ces enseignants, les stagiaires ont été invités à décrire leurs émotions, perceptions et réflexions au cours de ces différentes séquences d'observation pour en comprendre les effets formatifs. Les résultats montrent le potentiel d'apprentissage généré par ces dispositifs d'observation vidéo et in situ des situations professionnelles mais aussi la nécessité d'accompagner l'analyse réflexive des stagiaires pour qu'elle puisse contribuer à leur propre développement professionnel.

Les réformes des plans de formation des enseignants soulèvent de nombreuses difficultés à l'échelle internationale sans véritable consensus entre les chercheurs, les décideurs politiques et les responsables des plans de formation sur ce qui est le plus important à acquérir pour devenir enseignant (Schwille \& Dembélé, 2007). S'agit-il de viser prioritairement une formation universitaire de haut niveau adossée fortement aux savoirs scientifiques des disciplines scolaires à transmettre ou au contraire de viser une formation centrée sur l'acculturation précoce aux situations professionnelles? Cette alternative oppose une logique qui défend l'acquisition d'un savoir général produit par l'université à une logique d'acquisition directe sur le terrain (Merhan, Ronveaux \& Vanhulle, 2007).

Dans sa phase de rénovation de la formation des enseignants du second degré, la France a opté pour la première voie. Le processus en cours de mastérisation et d'universitarisation a pour ambition de garantir une formation académique de haut niveau, mais risque de priver par ailleurs les étudiants des réalités professionnelles du métier d'enseignant. Dès lors, la mise en place tout au long du cursus universitaire de stages ou de dispositifs d'observation du travail enseignant nécessite d'être repensée et optimisée pour ne pas renvoyer l'apprentissage du métier uniquement à la période d'exercice. Mais quel est l'impact réel de tels 
dispositifs? Que font les étudiants lorsqu'ils observent? A quoi pensent-ils? Qu'apprennent-ils? Quels dispositifs semblent les plus appropriés tout au long de leur formation?

\section{La rareté des études sur les effets des dispositifs de formation}

Pour Lortie (1975), l'apprentissage par observation du métier d'enseignant s'effectue tout au long de la scolarité des étudiants. Cette acculturation précoce et implicite aurait une incidence plus forte sur la manière d'enseigner des débutants que celle de leur formation initiale (Feiman-Nemser, 2001). Ce qui pose ici un problème essentiel pour l'observation en classe: comment faire en sorte que les stagiaires passent d'une observation habituée, sous-tendue par leurs connaissances d'anciens élèves, leurs croyances et habitudes liées à la forme scolaire, à une observation nouvelle, qui fasse rupture avec la première en ouvrant chez eux de nouveaux horizons?

Là encore, les dispositifs d'observation en classe doivent permettre d'opérer des conversions du regard des professeurs stagiaires pour appréhender les situations scolaires avec une autre intelligibilité. A ce titre, plusieurs scénarios de formation par l'observation entre professeurs débutants du second degré ont été évalués dans le cadre d'un dispositif de formation dans l'Académie de Créteil. Les résultats montrent l'indexation de leurs grilles de lecture de l'activité en classe à des critères d'efficacité pragmatique, et l'intérêt de la co-observation entre pairs dans la mesure où celle-ci prend des formes coopératives non concurrentielles du fait de la symétrie des statuts des participants (Ria, 2007). Cependant l'observation demeure une activité encore largement énigmatique lorsqu'elle est inscrite dans un parcours de formation.

Plus largement, les recherches portant sur les effets des ingénieries de formation sur la professionnalisation des étudiants se destinant à l'enseignement sont peu nombreuses. Celles portant sur la transformation des préoccupations des professeurs stagiaires au gré de leur formation (Serres \& Ria, 2007) ou encore sur l'évolution des normes professionnelles qu'adoptent les professeurs stagiaires du premier degré en cours de formation (Daguzon, 2009) ne procèdent pas à une analyse systématique des effets d'un dispositif ou d'une série de dispositifs sur leur développement professionnel. Quant aux évaluations des cursus universitaires, traduisant le taux global de satisfaction/insatisfaction des étudiants, elles ne donnent pas une idée précise des transformations que ces dispositifs de formation peuvent favoriser. Dès lors, l'élaboration de ressources pour la formation est fondée davantage sur des croyances ou «des heuristiques de bon sens» de la part des formateurs, parfois sur la prise en compte des critères de satisfaction des étudiants des sessions précédentes, que sur des programmes de recherche s'attachant à identifier et optimiser la pertinence des dispositifs selon des modalités de «conceptions continuées dans l'usage». 
Dans cet effort d'étude plus systématique des effets des dispositifs de formation, un nouveau courant de recherche en sciences humaines s'attache à repérer comment les acteurs apprennent avec et à partir des nouvelles technologies vidéo (Derry et al., 2009, sous presse). En effet, les multiples possibilités d'enregistrement, de stockage, de partage et d'étude font progressivement de ce média un artefact potentiellement fécond pour les environnements de formation. Des travaux exploratoires en France se sont intéressés aux effets, sur des enseignants du second degré en fin de formation initiale, de situations de simulation vidéo mettant en scène: a) des "pairs anonymes» (extrait de vidéo d'activités typiques de débutants), b) «soi-même» (extrait de vidéo de classe choisi par l'enseignant) et c) "son expérience» (extrait de vidéo de classe incluant le point de vue de l'acteur obtenu à partir d'un entretien d'autoconfrontation). Les premiers résultats montrent que l'activité d'observation y est vécue respectivement: a) de manière réconfortante, b) comme une épreuve difficile et c) comme révélatrice de l'expérience professionnelle à la fois singulière et typique (Leblanc $\&$ Ria, sous presse).

Cette contribution propose quant à elle de décrire et d'analyser un dispositif de formation destiné aux professeurs stagiaires de l'enseignement secondaire d'un institut de formation du centre de la France ${ }^{1}$ ayant pour vocation de les préparer à leur première affectation professionnelle. Ce dispositif, intitulé «Entrée dans le métier», est composé d'enseignements magistraux mobilisant des séquences vidéo et d'un stage en établissements scolaires relevant de l'éducation prioritaire $^{2}$, dans une académie d'accueil réputée difficile ${ }^{3}$, qu'ils découvrent pour une grande majorité lors de leur première année d'enseignement. Ce dispositif est sous-tendu par la conviction qu'il est possible «d'accélérer la professionnalisation» des étudiants en considérant l'activité professionnelle comme l'objet même de la formation à partir d'une démarche itérative qui articule: a) des observatoires ${ }^{4}$ de la pratique enseignante en situation réelle, telle qu'ils pourront la découvrir dès leur prochaine rentrée scolaire, b) la conception et la mise en œuvre de situations de formation indexées à cette réalité professionnelle et c) l'étude des effets de ces nouveaux dispositifs de formation sur les acteurs euxmêmes (Leblanc, Ria, Dieumegard, Serres \& Durand, 2008; Ria, Leblanc, Serres $\&$ Durand, 2006). Cette démarche s'alimente d'un double observatoire:

- d'une part, un observatoire de recherche $e^{5}$ sur le développement professionnel des néo-titulaires dans des établissements classés en éducation prioritaire de l'Académie de Créteil, permettant de comprendre leur travail quotidien, leur adaptation à l'ensemble des nouvelles prescriptions devant des publics très hétérogènes,

- d'autre part, un observatoire de l'activité en formation d'enseignants stagiaires confrontés à des situations de simulation vidéo construites sur la base d'un observatoire-conservatoire des pratiques professionnelles des enseignants débutants et expérimentés.

Dans ce dispositif «Entrée dans le métier», les stagiaires sont confrontés successivement à l'observation d'une série d'extraits vidéo de l'observatoire de Créteil 
complétée par des analyses produites par la recherche, et l'observation deux mois plus tard du travail d'enseignants exerçant dans les mêmes contextes professionnels que ceux présentés préalablement en conférence. Il s'agit moins de comparer les effets respectifs de deux dispositifs très différents, l'un en centre de formation à partir de supports vidéo et l'autre sur le terrain professionnel, que de comprendre la dynamique de transformation qu'ils procurent en les proposant successivement aux stagiaires comme un même espace de formation conçu selon des modalités et des empans temporels différents mais à partir d'options théoriques communes.

\section{Options théoriques de la conception des dispositifs de formation}

Ce dispositif de formation s'inscrit dans un programme de recherche scientifique et technologique en formation d'adultes prenant et spécifiant l'activité humaine comme objet théorique, d'étude et de conception dans une quête de pertinence écologique et scientifique (Durand, 2008). La conception de la formation professionnelle prend en compte le caractère complexe et évolutif du travail selon les visées de la didactique professionnelle dans une épistémologie énactive (Leblanc \& Ria, 2008), caractérisée par quatre présupposés essentiels: a) l'activité est indissociable de la situation dans laquelle elle prend forme et l'acteur participe à la construction de cette situation, b) elle s'accompagne d'une conscience préréflexive incorporée dans l'action, dont l'acteur peut rendre compte au moins en partie, moyennant des conditions favorables de collaboration, c) elle donne lieu à la construction de connaissances sur la base d'inférences validant ou invalidant des connaissances antérieures ou en construisant de nouvelles connaissances en relation avec l'efficacité pragmatique des actions réalisées dans la situation présente, d) elle exprime à la fois une singularité et une appartenance à une communauté.

La formation est conçue comme non prescriptive: le caractère d'autonomie et d'indétermination de l'activité implique de concevoir le processus d'influence formatrice et de transmission comme des perturbations potentielles du formé et non comme des prescriptions de son activité (Durand, Ria \& Veyrunes, 2009). Les dispositifs et moyens de la formation sont conçus comme des artefacts placés dans l'environnement des formés, qui peuvent ou non être significatifs pour eux, en fonction de leur culture, de leur engagement dans la situation, et de leur expérience et histoire personnelles. Autrement dit, c'est toujours le formé qui a l'initiative et cette influence formatrice potentielle peut s'opérer de diverses manières, notamment: a) en conférant à la situation scolaire une autre signification (des indices nouveaux dans la classe, de nouvelles préoccupations), b) en ouvrant le champ des possibles des formés (sans qu'une réalisation du possible ne se concrétise dans l'immédiat) et c) en contribuant à la validation, invalidation des 
savoir-faire déjà mobilisés, mais aussi à la construction de nouveaux savoir-faire que les enseignants vont pouvoir tester en classe.

De l'élaboration de situations de formation indexées à l'activité professionnelle réelle à l'analyse de l'activité en formation à partir de ces situations, cette conception itérative de la formation opère trois déplacements essentiels relativement à la conception traditionnelle des dispositifs de formation en France. Le premier déplacement consiste à passer d'une approche normative et figée de la formation à une approche située et développementale prenant en compte la diversité des situations et le temps de la construction de l'activité professionnelle en formation. Ceci suppose de décrire et analyser les mondes propres des formés comme un processus se déployant dans le temps et dans de multiples situations (Serres \& Ria, 2007). Le second déplacement consiste à ne pas réduire le processus de formation à l'acquisition et à la mise en œuvre de savoirs sous la forme de compétences mais à comprendre le développement de l'activité professionnelle selon une acception plus large (dépassant les seuls enjeux didactiques), notamment en pointant la dimension essentielle de participation et d'intégration à une communauté professionnelle. Le troisième déplacement consiste à décrire les cheminements progressifs vers l'expertise des débutants plutôt que de se focaliser sur la délimitation des pratiques reconnues pour leur expertise. L'hypothèse sousjacente est que l'activité experte peut être mobilisée en formation, non pas comme une référence unique, mais selon son degré de compatibilité avec l'activité débutante en cours de développement et son degré de pertinence compte tenu de la nature des situations professionnelles étudiées.

\section{Observation vidéo Lors d'une conférence en centre de formation}

\section{Objets de formation et méthode de recueil de données pendant la conférence}

La conférence destinée à 110 professeurs stagiaires de diverses disciplines scolaires du second degré, s'est appuyée sur une modélisation par la recherche des transformations typiques d'une des activités les plus problématiques pour les enseignants débutant en milieu difficile ${ }^{6}$ : l'accueil et la mise au travail de leurs élèves. Trois caractéristiques principales ont été mises en avant lors de la présentation d'une dizaine d'extraits vidéo hiérarchisés les uns par rapport aux autres selon des critères d'efficacité subjectifs et objectifs': a) l'évolution de l'engagement corporel et intentionnel des néo-titulaires en début de cours, b) la mobilisation de plus en plus rapide des savoirs scolaires, notamment sous la forme écrite, pour enrôler les élèves et c) la construction empirique de différentes configurations collectives stables et régulières structurant l'activité des élèves et offrant de nouvelles opportunités pour leurs propres actions (pour plus de détails, Ria 2009). 
Le visionnage de chacun des extraits vidéo a été complété systématiquement par l'explicitation du sens que les enseignants conféraient à leur propre activité (émotions, préoccupations, indices perceptifs, connaissances...) pour éviter toute interprétation abusive des seuls comportements observés. Les analyses et interventions du formateur ont porté sur la nécessité de concevoir l'activité des débutants: a) dans une perspective dynamique, c'est-à-dire en l'appréhendant comme une forme provisoire, partiellement stabilisée, partiellement satisfaisante, et dont la modélisation des autres activités typiques en amont et en aval de celle-ci donne des clefs pour la comprendre et la transformer, b) dans ses aspects les plus génériques d'une communauté débutante en cours de développement, et non selon les difficultés inhérentes à des enseignants en particulier et c) dans son chemin vers l'expertise et non comme un produit dégradé, ou seulement caractérisé en creux, en référence à l'activité experte. Dans cette perspective, la modélisation de l'activité débutante proposée en formation était appréhendée comme un guide orientant l'observation et l'interprétation des stagiaires.

Un questionnaire de quatre pages a été distribué et présenté au début de la conférence à l'ensemble des professeurs stagiaires (Tableau 1). Il permettait à chaque stagiaire d'indiquer le confort-inconfort vécu sur une échelle ordinale en sept points (de -3 très inconfortable à +3 très confortable) lors du visionnage de chaque extrait vidéo, mais aussi de documenter complémentairement ses réactions et interprétations, les expériences personnelles qui émergeaient pendant le visionnage, et enfin les commentaires ou réflexions ayant émergé lors de l'analyse de la vidéo par le formateur ${ }^{8}$. Deux chercheurs ont sollicité quatre professeurs stagiaires volontaires pour recueillir à la volée des commentaires oraux lors de la conférence, en parlant à voix basse pour limiter les perturbations et ont procédé avec ces mêmes stagiaires à des entretiens semi-directifs pour identifier quelques mois après les effets du dispositif «Entrée dans le métier» sur le développement de leur activité professionnelle.

\section{Effets sur les professeurs stagiaires de l'observation d'extraits vidéo}

Le panel d'extraits vidéo présentait d'abord aux professeurs stagiaires des situations d'accueil des élèves non contrôlées puis de plus en plus maîtrisées. Il ne s'agissait pas de montrer des enseignants en difficulté mais les difficultés typiques des débutants dont la modélisation par la recherche permettait d'en comprendre la nature et les transformations progressives. Le visionnage d'une jeune enseignante attendant durant plusieurs minutes le silence des élèves en début de cours a généré chez les professeurs stagiaires de l'inconfort: moyenne de -0.8 sur l'échelle de $-3 /+3$. Une petite minorité d'entre eux (moins de $10 \%$ ) a indiqué -3 lors du visionnage avec des réactions montrant à quel point cet extrait vidéo les a conduits à réinterroger la pertinence de leur choix professionnel: «Vivre de telles situations m'amène à me demander si je suis fait pour le métier. Ces situations usent notre patience lorsqu'elles sont récurrentes dans la journée...». 
Tableau 1: Modalités d'organisation du dispositif "Entrée dans le métier» et de recueil de données

\begin{tabular}{|c|c|c|c|}
\hline \multicolumn{2}{|c|}{ Observation vidéo [conférence] } & \multicolumn{2}{|c|}{ Observation in situ [établissements] } \\
\hline $\begin{array}{l}110 \text { professeurs } \\
\text { stagiaires du second } \\
\text { degré }\end{array}$ & $\begin{array}{l}\text { Conférence plénière en } \\
\text { centre de formation } \\
\text { (novembre 2008) }\end{array}$ & $\begin{array}{l}40 \text { professeurs stagiaires } \\
\text { volontaires parmi les } \\
110 \text { ayant assisté à la } \\
\text { conférence }\end{array}$ & $\begin{array}{l}\text { Stage d'observation de } 3 \\
\text { jours dans des établisse- } \\
\text { ments éducation priori- } \\
\text { taire en banlieue pari- } \\
\text { sienne (janvier 2009) }\end{array}$ \\
\hline & $\begin{array}{l}\text { Visionnement de } 8 \text { cas } \\
\text { vidéo hiérarchisés et } \\
\text { relatifs à une même } \\
\text { situation professionnelle } \\
\text { problématique }\end{array}$ & & $\begin{array}{l}12 \text { heures d'observation } \\
\text { du travail enseignant } \\
\text { dans plusieurs disci- } \\
\text { plines scolaires et } \\
\text { échanges avec l'équipe } \\
\text { éducative }\end{array}$ \\
\hline & $\begin{array}{l}\text { Analyse des cas vidéo } \\
\text { par le formateur }\end{array}$ & & $\begin{array}{l}\text { Débriefings avec les } \\
\text { enseignants observés } \\
\text { puis avec des formateurs }\end{array}$ \\
\hline \multicolumn{2}{|l|}{ Recueil de données } & \multicolumn{2}{|l|}{ Recueil de données } \\
\hline \multirow[t]{2}{*}{$\begin{array}{l}\text { Questionnaires aux } 110 \\
\text { professeurs stagiaires }\end{array}$} & $\begin{array}{l}\text { Evaluation du vécu } \\
\text { émotionnel pendant le } \\
\text { visionnement des cas } \\
\text { vidéo }\end{array}$ & $\begin{array}{l}\text { Questionnaires aux } 40 \\
\text { professeurs stagiaires } \\
\text { pendant le stage d'obser- } \\
\text { vation }\end{array}$ & $\begin{array}{l}\text { Recueil des réactions et } \\
\text { analyses à la suite des } \\
\text { observations in situ et } \\
\text { des échanges dans l'éta- } \\
\text { blissement }\end{array}$ \\
\hline & $\begin{array}{l}\text { Recueil des commen- } \\
\text { taires et analyses avant et } \\
\text { après l'intervention du } \\
\text { formateur }\end{array}$ & & \\
\hline $\begin{array}{l}\text { Entretiens à la volée de } \\
4 \text { professeurs stagiaires } \\
\text { volontaires pendant la } \\
\text { conférence }\end{array}$ & $\begin{array}{l}\text { Enregistrement audio } \\
\text { des commentaires et } \\
\text { analyses des } 4 \text { stagiaires } \\
\text { pendant la conférence }\end{array}$ & $\begin{array}{l}\text { Entretiens semi-directifs } \\
\text { avec les } 4 \text { mêmes profes- } \\
\text { seurs stagiaires un mois } \\
\text { après le stage }\end{array}$ & $\begin{array}{l}\text { Effets du dispositif de } \\
\text { formation "Entrée dans } \\
\text { le métier" dans son } \\
\text { ensemble sur leur propre } \\
\text { activité professionnelle }\end{array}$ \\
\hline
\end{tabular}

L'observation a généré une forte empathie (entendue comme conscience de la souffrance de l'autre) à l'égard de l'enseignante ne parvenant pas à obtenir le silence en début de cours, et parfois même de la compassion (entendue comme souffrance avec l'autre): «Quand je vois cette vidéo, je me dis qu'elle est submergée et de fait, je le suis aussi. C'est insoutenable de voir cela, c'est absolument inconfortable! Ça m'interroge sur ma façon d'imposer ma présence en classe». Les stagiaires ont majoritairement interprété la situation d'attente comme une épreuve pour l'enseignante, alors que celle-ci, lors de l'entretien avec le chercheur, a au contraire signifié le caractère ordinaire et sans appréhension particulière de son expérience lors du début du cours observé. L'expression d'un vécu non congruent avec les premières intuitions des stagiaires a favorisé chez eux de nouvelles interprétations: "C'est sûr que je réagis par rapport à mes propres classes... Vu que c'est sa deuxième année dans l'établissement, elle a dû vivre des choses plus cuisantes tout au début, là, elle relativise compte tenu de son 
passé...». Seulement deux ou trois stagiaires ont vécu la séquence vidéo de manière très confortable $[+3]$. Ils apparaissaient davantage dans une posture réflexive que les observateurs ayant éprouvé des affects négatifs: «Elle ne perd pas patience dans cette situation que j'estime plus ou moins normale en début de cours. Cette situation s'apparente à certaines entrées en classe dans le lycée où je suis stagiaire. En voyant cette vidéo je me dis que c'est intéressant de ne pas s'énerver, de persister, de ne pas perdre patience...».

Ces premières observations ont favorisé des procédures d'invalidation de la pertinence des registres d'intervention mobilisés par les enseignants en classe: «Je me demande si le dialogue en début de cours est la meilleure façon d'instaurer le calme». Le visionnage de deux vidéos d'enseignants débutants plus efficaces dans leurs débuts de cours a été vécu de manière plus confortable [moyennes respectivement de +0.9 et +1 ], mais toujours avec de la compassion à l'égard de ces jeunes enseignants déployant beaucoup d'énergie pour tenir leurs classes. Les vidéos de l'activité d'enseignants plus chevronnés et efficaces en début de cours ont favorisé l'émergence d'émotions très positives lors du visionnage [moyennes de +2.5 et +2.2 ]. Ils ont reconnu l'intérêt de dépasser la seule observation vidéo pour comprendre, à partir des entretiens d'autoconfrontation, comment leur activité s'était construite. Ils ont noté sur leur fiche des mots clefs, des attitudes ou postures (regards, usage des intonations de la voix, attitudes théâtralisées, etc.), des pistes pour leurs propres actions et des expressions ou métaphores employées par les enseignants dans les entretiens pour évoquer leur activité, comme par exemple: «La construction du silence en début de cours comme une nappe pouvant se propager et se solidifier dans la classe».

Soixante-dix pour cent des 110 stagiaires ont estimé que le visionnage de l'observatoire du travail d'enseignants en milieu difficile leur a procuré des pistes concrètes pour leur pratique professionnelle actuelle, mais aussi future. Ils ont procédé à de nombreux va-et-vient entre leurs vécus et les ressources vidéo, et ce malgré les différences de contextes d'intervention: "Cette vidéo me rappelle mes premières semaines de cours». Si des activités mimétiques se sont déployées lors du visionnage des extraits vidéo des débutants: "Je suis comme elle», les observateurs se sont parfois même identifiés aux plus expérimentés: "Je suis un petit peu comme elle dans le sens où je ne vais pas m'imposer comme ça par la simple présence, c'est plus mon calme et ma manière d'être qui vont faire que je vais pouvoir avancer...». Ces séquences vidéo et leurs analyses ont favorisé l'émergence d'analyses de pratiques privées: «J'ai pu repérer mes propres comportements, mes habitudes... Je me sens en mesure de les remettre en question, de mesurer les points faibles et les points forts». Comme si l'observation de l'activité d'un tiers pouvait servir de révélateur (de catalyseur) pour sa propre pratique: "J'ai pu repérer certaines de mes attitudes qui m'auraient pris seul des années à déconstruire», et comme si la confrontation à une diversité d'activités typiques d'enseignants débutants plus ou moins efficaces leur ouvrait des possibles qu'ils ne se seraient pas autorisés à mobiliser spontanément: "Je vois de nouvelles pistes possibles 
dans lesquelles je ne me projetais pas et qui deviennent moins inaccessibles, peut être même envisageables...».

Les stagiaires à l'issue de cette conférence ont estimé ne pas être du tout rassurés $(15 \%)$, un peu rassurés (39\%), plutôt rassurés $(38 \%)$, beaucoup rassurés (8\%) quant à la possibilité d'être mutés dans un établissement de milieu difficile l'année suivante. Pour 90\% d'entre eux, les extraits vidéo et leurs analyses ont permis de mieux comprendre les formes d'adaptation possibles de l'activité enseignante. Ce qui aux yeux de plusieurs stagiaires permettait de remettre en cause une croyance résistante, énoncée tout au long de leur formation, sur le déterminisme très fort voire irréversible des premiers gestes professionnels dans la conduite de leurs classes: «En fait toutes ces vidéos permettent de voir que les profs peuvent changer de tactiques, de méthodes avec leurs élèves quand ça marche pas et c'est plus rassurant que le discours un peu "formaté» de la formation qui dit que si l'on rate le début [de l'année avec les élèves] cela peut être difficile de récupérer sa classe... Ici, on voit des enseignants qui sont capables en fait de se remettre en cause et de trouver d'autres méthodes pour assurer leurs cours».

\section{Observation in situ lors d'un stage d'immersion professionnelle}

Deux mois plus tard, une quarantaine de professeurs stagiaires, ayant assisté à la conférence portant sur l'enseignement en milieu difficile, ont participé durant trois jours à des séquences d'observation de l'activité en classe d'enseignants néotitulaires et d'enseignants plus chevronnés dans plusieurs disciplines scolaires au sein d'établissements classés en éducation prioritaire et situés dans la ceinture parisienne. Ils ont procédé ensuite à des débriefings post-observation avec les intervenants et les formateurs et assisté à des échanges avec différents acteurs de l'établissement (chefs d'établissement, conseillers principaux d'éducation, etc.). Lors de ce stage, les professeurs stagiaires ont ressenti des émotions très fortes, sans aucune comparaison avec celles éprouvées dans l'amphithéâtre: «Je suis partie vraiment terrorisée...»9 . Cette immersion professionnelle a été vécue pour certains comme une épreuve probatoire cruciale: «J'avais besoin de me projeter, d'aller voir réellement ce qui m'attend l'année prochaine, me rassurer quant à ma capacité à prendre en main une classe difficile, observer quels aménagements sont possibles dans ce genre de contexte...».

L'observation a largement débordé le cadre des activités de classe: "J'ai été marquée par un drôle de sentiment suscité par l'observation d'une population d'élèves presque uniquement constituée d'individus issus de l'immigration face à une population d'enseignants complètement "étrangère» au quartier, à la cité, se déversant par le métro chaque matin et repartant le soir... Quelque chose d'un peu dérangeant...». Les professeurs stagiaires ont spontanément comparé les différents contextes d'enseignement: "J'ai vraiment découvert un autre monde, 
incomparable avec celui dans lequel je fais mon stage depuis le début de l'année: des élèves livrés à eux-mêmes dans la cour, beaucoup d'agitation, une violence palpable, latente...». La comparaison s'est poursuivie pour certains au retour de leur stage: «En revenant dans mon collège, j’ai été marquée à rebours par le décalage énorme concernant les seuils de tolérance entre les règles de vie scolaire de mon établissement et celles dans le collège parisien: ici, les élèves sont presque rangés au cordeau dans la cour...» ${ }^{10}$. Une fois de plus, une petite minorité a vécu le stage avec une plus grande neutralité: "J'ai vécu ce stage avec une certaine distance... je ne retrouvais pas mes propres élèves et donc je ne me suis pas projeté affectivement, n'ai pas fait de comparaison systématique avec mes élèves, de comparaison entre les deux contextes d'enseignement. J'ai observé en pensant à l'année prochaine pour m'habituer à ces élèves, m'imprégner des ambiances et voir ce qui marche pour les tenir et tenir soi-même...".

La conférence en centre de formation a participé à l'orientation des premières observations, parfois de manière inattendue: "Dès la première heure en classe, j'ai cherché à retrouver ce qu'on nous avait raconté sur les façons d'entrer en classe, de se servir de l'écrit pour mettre les élèves au travail, et en fait... Je fus super surprise de voir que le cours observé ressemblait comme deux gouttes d'eau à ce que je vis moi-même, enfin... avec des élèves moins durs...». Les débriefings post-observation ont permis aux professeurs stagiaires de rendre compte de moments d'observation marquants, en tentant d'identifier les principaux organisateurs de l'activité professionnelle: "J'ai été hyper impressionné par une enseignante en sciences de la vie et de la terre: la construction du sens de sa discipline, sa façon de démontrer aux élèves l'exactitude ou non d'un raisonnement scientifique, la sensation d'un cadre fluide, clair et ferme à la fois... Elle parvient à être exigeante en termes de contenus d'enseignement, de vocabulaire employé tout en créant une atmosphère calme, douce avec humour et bienveillance...». Au fil des observations de classe et des interactions avec les enseignants observés, les professeurs stagiaires ont interrogé la nature des critères de l'efficacité professionnelle dans ce genre d'établissements: «Ce stage m'a fait prendre conscience qu'un enseignement ferme et de qualité est possible avec ces jeunes défavorisés. Pour obtenir le contrôle de la classe, il faut tenir ses engagements et ne rien laisser passer, être exigeant avec soi-même et les élèves... Plusieurs profils d'enseignants très différents peuvent être efficaces; en fait, il n'y a pas qu'une seule façon de faire... Il est possible d'être très efficace avec ce genre de public sans avoir forcément une très grande expérience professionnelle».

Finalement s'est opéré chez les professeurs stagiaires un profond soulagement à la suite de la découverte d'un environnement professionnel trop souvent stigmatisé par les médias: «Ce stage démystifie les a priori concernant l'enseignement en milieu difficile: forte cohésion et solidarité des membres de l'équipe éducative, les situations très délicates à gérer avec les élèves restent marginales... J'ai l'impression d'avoir moins peur de ces élèves-là maintenant... Il est possible d'aborder des contenus d'enseignement exigeants avec ce type de public: les élè- 
ves en sont même demandeurs...». Plusieurs d'entre eux ont réinterrogé leurs propres pratiques professionnelles et ont décidé d'en infléchir l'orientation dès le retour du stage d'observation: "J'ai vraiment pris conscience que crier ne servait à rien dans ce contexte difficile, on peut s'épuiser sans aucune efficacité... Avec mes petits sixièmes [stage en responsabilité durant une année scolaire], j’ai conscience de crier bien trop... Au retour du stage de Créteil, j’ai changé ma relation avec eux en tentant d'adopter une attitude exigeante mais dans une relation plus apaisée» 11 .

\section{Discussion générale}

\section{Des dispositifs d'observation pour apprendre des situations professionnelles}

L'une des difficultés de la formation initiale des enseignants du second degré en France réside dans le fait de ne pas pouvoir les préparer concrètement aux difficultés de leur futur métier, s'exerçant le plus souvent dans des conditions plus dures que celles de leurs stages de formation initiale. Cette méconnaissance de l'horizon professionnel des académies d'accueil est à l'origine chez eux de fortes inquiétudes et d'un véritable choc lors des premières semaines d'enseignement. La conception de dispositifs d'observation du travail enseignant dans ces établissements permet "d'apprendre - à distance - des situations professionnelles» (Pastré, 2005); cette «expérience sans risque» participe à la construction identitaire des professeurs stagiaires, mais aussi au développement de leur part d'une réflexion critique quant aux gestes professionnels les plus adéquats pour intervenir face à des publics hétérogènes. Un dispositif de ressources vidéo peut constituer une première étape de sensibilisation aux difficultés typiques de l'agir professionnel, un stage d'observation in situ une seconde étape d'immersion dans l'environnement scolaire et d'acculturation professionnelle. Dans ces deux dispositifs, l'observation n'a pas la même fonction et les mêmes effets.

L'observation en centre de formation de situations professionnelles problématiques ou critiques pour les débutants à partir d'extraits vidéo permet aux professeurs stagiaires de procéder "par procuration» à des anticipations, pour éviter qu'ils ne découvrent seuls la réalité d'un métier. L'observation de l'activité de pairs prend ici tout son sens. La recension par la recherche des "passages à risques» des néo-titulaires (Rayou \& Ria, 2009) a pour première fonction de déculpabiliser les stagiaires vis-à-vis des difficultés typiques de la communauté débutante. Le dispositif de ressources vidéo permet ensuite de comprendre la dynamique de transformation de l'activité lors d'un même moment critique. A cette étape, l'observation des modalités d'adaptation de pairs ou d'enseignants expérimentés plus efficaces devient pertinente. Mais la compréhension de cette dynamique de transformation ne va pas de soi; elle doit être guidée en formation par des critères explicites de catégorisation de l'activité. 
L'observation en immersion constitue pour les professeurs stagiaires la première expérience d'entrée dans la communauté éducative de leur futur métier: au-delà des observations cliniques, les échanges informels et les connivences avec les collègues participent à leur très fort besoin de réassurance et de reconnaissance identitaire. Mais peu à peu, et grâce au travail d'observation amorcé préalablement en centre de formation et aux débriefings post-observation, ils parviennent à analyser les situations professionnelles observées avec une plus grande acuité et à identifier de manière plus systématique les régularités des modalités d'agir dans ces environnements scolaires.

Dans les deux dispositifs étudiés, l'observation constitue le début d'un processus d'acculturation professionnelle étayé par le sens que les enseignants conferent à leurs propres activités (entretiens d'autoconfrontation pour l'observation vidéo et débriefings post-observation pour l'observation in situ) mais aussi guidé par les analyses du formateur. Les professeurs stagiaires apprennent par ces dispositifs d'observation à convoquer et à analyser leurs propres expériences professionnelles sur la base d'indices typiques de situations de travail qu'ils reconnaissent sur vidéo ou en présentiel. Ils apprennent aussi à identifier des gestes professionnels "pris sur le vif», dont l'efficacité pratique sur les comportements en classe les incite à les tester eux-mêmes avec leurs propres élèves.

\section{Observation participante, observation mimétique et fictionnelle}

Les résultats obtenus lors de cette première étude des effets de dispositifs d'observation nécessitent d'être complétés par d'autres données. Néanmoins, ce premier corpus montre que l'observation en formation ne peut se suffire à ellemême. La réalité observée ne s'appréhende pas de manière directe, elle est toujours liée à ce qui «fait signe» dans l'environnement. L'observation peut s'avérer relativement pauvre si les professeurs stagiaires ne parviennent pas à s'extirper de leur monde familier pour en tirer quelques enseignements nouveaux. Sans prise de distance, sans effort pour rendre étranger le familier, aucune forme particulière et nouvelle n'émerge: ils revivent les situations telles qu'ils les ont vécues en tant qu'élèves. Il peut être alors difficile voire ennuyeux de prolonger cette observation peu discriminante et donc peu instructive. L'offre en formation d'une modélisation des situations professionnelles les plus cruciales pour l'exercice du métier constitue une entrée prometteuse pour viser chez les professeurs stagiaires des processus d'apprentissage authentiques. Deux modalités d'observation apparaissent chez eux de manière typique.

Premièrement, l'observation, qu'elle soit réalisée à partir d'un support vidéo ou en situation de classe, peut favoriser une observation-vécue ou participante: les événements observés en classe "transportent» l'observateur hors de lui-même, le projettent à la place de l'autre. Il s'estime affecté, touché par la situation vécue tout aussi pleinement que l'observé. La compassion éprouvée limite l'interprétation des situations observées. Le temps de l'observation ne correspond plus à un 
temps chronologique objectif mais à un «temps-vécu» avec une forte adhérence aux événements. La dualité observateur-observé disparait. L'accompagnement de l'observation peut permettre de transformer les phénomènes de compassion en des attitudes empathiques davantage enclines à favoriser la réévaluation et/ou à la re-légitimisation d'expériences de travail vécues par les professeurs stagiaires jusque-là de manière insatisfaisante voire même culpabilisante.

Deuxièmement, l'observation peut progressivement permettre un jeu de comparaison des ressemblances et dissemblances entre des indices, des actions, des effets communs ou au contraire différents de l'activité professionnelle. Cette comparaison systématique permet de connaître une autre activité tout en reconnaissant la leur au moins ponctuellement et partiellement. Ces allers-retours entre eux-mêmes et autrui conferent à leur propre activité un caractère d'étrangeté, d'extériorité favorisant la connaissance de soi et la remise en cause de leurs propres repères pour enseigner. Ces mouvements comparatifs participent à la construction de fictions pour soi-même, entre sa propre réalité et le «comme si» permis par les simulations vidéo grâce notamment à l'euphémisation des conséquences des actions, à la possibilité de revivre «l'expérience fictionnelle» de nombreuses fois et à la tolérance à l'erreur. Ils conduisent à trouver un compromis efficace entre l'implication dans la situation favorisée par «l'immersion mimétique» (Schaeffer, 1999) et la distanciation nécessaire pour apprendre et se développer en faisant un pas de côté indispensable.

Finalement, l'observation à distance ou en immersion au sein des situations professionnelles ne remplace aucunement l'expérience réelle mais la prépare. Elle permet de construire des liens de signification entre deux mondes qui s'ignorent encore trop souvent (celui de la formation initiale dans les instituts de province et celui de l'exercice professionnel dans les académies d'accueil) en identifiant des éléments génériques de l'exercice professionnel au-delà des particularités locales. Les modélisations et analyses de l'activité enseignante proposées par la recherche peuvent ainsi constituer pour les professeurs en formation des instruments d'orientation de leur développement professionnel et pour les formateurs des ressources pour les aider à renouveler leur accompagnement en anticipant ce "passage à risque» que demeure l'entrée dans le métier.

\section{Notes}

1 IUFM d'Auvergne, Clermont Ferrand.

2 Initiée en 1981, la politique d'éducation prioritaire a pour objet de renforcer l'action éducative dans les zones où les conditions sociales constituent un obstacle pour la réussite scolaire des enfants et adolescents qui y vivent.

3 L'Académie de Créteil accueille chaque année plus de 1500 néo-titulaires du second degré et se caractérise par la très forte hétérogénéité de ses populations scolaires sur les plans de leur scolarisation et socialisation.

4 Un observatoire mobilise un outillage théorique et méthodologique adéquat pour recueillir et interpréter les processus ou phénomènes constitutifs de l'activité étudiée.

5 Dans le cadre du programme scientifique «Professionnalité Enseignante» de l'Institut National de Recherche Pédagogique. 
6 D’autres épisodes critiques pour les débutants peuvent constituer des ressources en formation: la maîtrise d'élèves perturbateurs, la délivrance de consignes, l'explication d'une notion abstraite suscitant des polémiques, la canalisation de l'activité liée au travail de groupes, le contrôle des élèves dans un espace ouvert, etc.

7 Les critères subjectifs correspondant à l'expérience appréhendée du point de vue de l'enseignant et les critères objectifs aux effets de l'activité de l'enseignant sur les comportements des élèves, sur l'ambiance de la classe.

8 La conférence a été interrompue à plusieurs reprises quelques minutes pour donner aux professeurs stagiaires le temps nécessaire à l'écriture de leurs commentaires.

9 Un questionnaire a été de nouveau proposé aux 40 professeurs stagiaires pour qu'ils puissent décrire leurs expériences lors du stage de trois jours et plusieurs situations d'observation en classe, suivies de commentaires et d'analyses personnelles.

10 Verbatim extrait d'un entretien semi-directif post-stage conduit un mois après le stage d'observation.

11 Verbatim extrait d'un entretien semi-directif post-stage.

\section{Références bibliographiques}

Daguzon, M. (2009). Formation initiale des professeurs des écoles: De la prescription à la redéfinition de la tâche (étude longitudinale). In R. Goigoux, L. Ria \& M.C. ToczekCapelle (Éd.), Les parcours de formation des enseignants débutants (pp. 101-120). ClermontFerrand: Presses Universitaires de Blaise Pascal.

Derry, S. J., Pea, R. D., Barron, B., Engle, R. A., Erickson, F., Goldman, R., Hall, R., Koschmann, T., Lemke, J. L., Sherin, G. M. \& Sherin, B. L. (2009, sous presse). Guidelines for conducting video research in the learning sciences. The Journal of the Learning Sciences.

Durand, M. (2008). Un programme de recherche technologique en formation des adultes. Une approche énactive de l'activité humaine et l'accompagnement de son apprentissage développement. Education et Didactique, 2, (2), 69-93.

Durand, M., Ria, L. \& Veyrunes, P. (2009). Analyse du travail et formation: Un programme de recherche empirique et technologique portant sur la signification et l'organisation de l'activité des enseignants. In F. Saussez (Éd.), Analyser l'activité enseignante: Des outils méthodologiques et théoriques pour l'intervention et la formation (pp. 137-157). Québec: Presses de l'Université de Laval.

Feiman-Nemser, S. (2001). From preparation to practice: Designing a continuum to strengthen and sustain teaching. Teacher College Record, 103, 1013-1055.

Leblanc, S. \& Ria, L. (2008). Observatoire de l'évolution de la professionnalité enseignante et dispositifs de formation de simulation vidéo. In G. Baillat \& D. Nicot (Éd.), Recherche sur la formation des enseignants en Europe: Contextes, nouveaux dispositifs, nouvelles pratiques.

Leblanc, S., Ria, L., Dieumegard, G., Serres, G. \& Durand, M. (2008). Concevoir des dispositifs de formation professionnelle des enseignants à partir de l'analyse de l'activité dans une approche énactive. Activités, 5, (1), 58-78.

Lortie, D. (1975). Schoolteacher: A sociological study. University of Chicago Press.

Merhan, F., Ronveaux, C. \& Vanhulle, S. (2007). Alternances en formation. Bruxelles: De Boeck.

Pastré, P. (2005). Apprendre par la simulation: De l'analyse du travail aux apprentissages professionnels. Toulouse: Octarès.

Rayou, P. \& Ria, L. (2009). Former les nouveaux enseignants. Autour des statuts, de l'organisation et des savoirs professionnels. Education et Sociétés, 23, (1), 79-90.

Ria, L. (2007). Transformation de l'activité professorale lors d'un dispositif d'observations entre pairs: Un enjeu de recherche et de formation pour l'accompagnement dans l'entrée dans le métier des enseignants du second degré en France. Formation et pratiques d'ensei- 
gnement en question. Revue des HEP de Suisse romande et du Tessin, 6, 77-98.

Ria, L. (2009). De l'analyse de l'activité des enseignants débutants en milieu difficile à la conception de dispositifs de formation. In M. Durand \& L. Filliettaz (Éd.), La place du travail dans la formation des adultes (pp. 217-243). Paris: PUF.

Ria, L., Leblanc, S., Serres, G. \& Durand, M. (2006). Recherche et formation en «analyse des pratiques»: Un exemple d'articulation. Recherche et Formation, 51, 43-56.

Schaeffer, J. M. (1999). Pourquoi la fiction? Paris: Seuil.

Schwille, J. \& Dembélé, M. (2007). Former des enseignants: Politiques et pratiques. Principes de la planification de l'éducation (Numéro 84). Paris: UNESCO.

Serres, G. \& Ria, L. (2007). Questionner la formation des enseignants à partir de la description des trajectoires des formés. Formation et pratiques d'enseignement en question. Revue des HEP de Suisse romande et du Tessin, 6, 99-119.

Mots clés: Enseignants débutants, développement professionnel, dispositifs de formation, effets de l'observation, didactique professionnelle

\section{Von der Video-Observation zur Observation im Feld: Eine Wirkungsstudie zur Unterrichtstätigkeit von Praktikanten in einem herausfordernden Milieu}

\section{Zusammenfassung}

Dieser Artikel beschreibt und analysiert ein Beobachtungsinstrumentarium, das für Praktikantinnen und Praktikanten einer französischen Lehrerbildungsinstitution für die Sekundarstufe geschaffen wurde. Mit Hilfe dieses Instrumentariums sollen die Studierenden auf den ersten Feldkontakt vorbereitet werden: Anhand der sukzessiv in den Unterricht eingeführten Videosequenzen zur Unterrichtsarbeit in einem schwierigen Milieu und der realen Beobachtungsvorgängen werden die Praktikantinnen und Praktikanten aufgefordert, ihre Emotionen, Erfahrungen und Reflexionen zu diesen unterschiedlichen Beobachtungssequenzen zu beschreiben. Ziel dieser Beobachtungswiedergabe ist es die eigenen Lerneffekte zu reflektieren. Die Resultate zeigen das Potential von Lerngefässen mit Video- und Unterrichtsbeobachtung. Sie verweisen aber auch auf die Wichtigkeit der reflexiven Begleitung der Studierenden, damit die Beobachtungen zur beruflichen Entwicklung beitragen können.

Schlagworte: Berufseinsteigende, Professionalisierung, Ausbildungskonzept, Beobachtungseffekte, Didaktik 


\section{Dall'osservazione video all'osservazione sul campo dell'attività d'insegnamento in condizioni difficili : effetti sui docenti in formazione}

\section{Riassunto}

L'articolo descrive gli effetti di un dispositivo di osservazione dell'attività di insegnanti in formazione di un istituto di formazione di livello secondario in Francia. Agli insegnanti, che osservano dapprima una serie di registrazioni video di attività d'insegnamento in condizioni difficili e che poi vengono confrontati, due mesi più tardi, con delle situazioni reali, si chiede di descrivere le proprie emozioni, percezioni e riflessioni al fine di identificare gli effetti formativi dell'esperienza. I risultati mostrano il potenziale derivante sia dalle osservazioni delle videoregistrazioni che da quelle in situazione, ma evidenziano anche la necessità di accompagnamento, affinché l'analisi riflessiva possa contribuire allo sviluppo professionale.

Parole chiave: Insegnamento dei principianti, sviluppo professionale, dispositivo di formazione, effetti dell'osservazione, didattica professionale

\section{From video observation to in situ observation of teachers' work in difficult situations: a study of effects on students teachers}

\section{Summary}

The article describes and analyzes the effects of the observation phase used during the training course run by a French secondary teacher's education and training institute. This observation phase is aimed at helping students to prepare for their first teaching job.

Students are successively confronted with difficult teaching situations first by watching them on video, them by observing them in situ. In order to foster their own learning process, students are then invited to describe their emotions, perceptions and reflections on the different observations made of their performances. Results show that there is potential for students to learn through this observation phase; but they also reveal the necessity of honing the students' analytical skills in order for this phase to contribute significantly to their professional development.

Key words: Career entry, professional skills, learning tool, observation effects, didactics 